\title{
Lipidomic Profiling Identifies Signatures of Poor Cardiovascular Health
}

\author{
Irma Magaly Rivas Serna ${ }^{1}$, Michal Sitina ${ }^{1}$, Gorazd B. Stokin ${ }^{1}$, Jose R. Medina-Inojosa ${ }^{2,3}{ }^{(1)}$, \\ Francisco Lopez-Jimenez ${ }^{2}$, Juan P. Gonzalez-Rivas ${ }^{1,4}\left(\mathbb{D}\right.$ and Manlio Vinciguerra ${ }^{1, *(1)}$
}

1 International Clinical Research Center (ICRC), St Anne's University Hospital, 53, 65691 Brno, Czech Republic; rivasser@ualberta.ca (I.M.R.S.); michal.sitina@fnusa.cz (M.S.); gorazd.stokin@fnusa.cz (G.B.S.); juan.gonzalez@fnusa.cz (J.P.G.-R.)

2 Division of Preventive Cardiology, Department of Cardiovascular Medicine, Mayo Clinic, Rochester, MN 55902, USA; medinainojosa.jose@mayo.edu (J.R.M.-I.); lopez@mayo.edu (F.L.-J.)

3 Marriot Heart Disease Research Program, Rochester, MN 55902, USA

4 Department of Global Health and Population, Harvard T.H. Chan School of Public Health, Boston, MA 02115, USA

* Correspondence: manlio.vinciguerra@fnusa.cz

Citation: Rivas Serna, I.M.; Sitina, M.; Stokin, G.B.; Medina-Inojosa, J.R.; Lopez-Jimenez, F.; Gonzalez-Rivas, J.P.; Vinciguerra, M. Lipidomic Profiling Identifies Signatures of Poor Cardiovascular Health. Metabolites 2021, 11, 747. https://doi.org/ 10.3390/metabo11110747

Academic Editor: Wilfried Le Goff

Received: 10 October 2021

Accepted: 27 October 2021

Published: 29 October 202

Publisher's Note: MDPI stays neutral with regard to jurisdictional claims in published maps and institutional affiliations.

Copyright: (c) 2021 by the authors. Licensee MDPI, Basel, Switzerland. This article is an open access article distributed under the terms and conditions of the Creative Commons Attribution (CC BY) license (https:// creativecommons.org/licenses/by/ $4.0 /)$.

\begin{abstract}
Ideal cardiovascular health $(\mathrm{CVH})$ is defined for the presence of ideal behavioral and health metrics known to prevent cardiovascular disease (CVD). The association of circulatory phospho- and sphingo-lipids to primary reduction in cardiovascular risk is unclear. Our aim was to determine the association of $\mathrm{CVH}$ metrics with the circulating lipid profile of a population-based cohort. Serum sphingolipid and phospholipid species were extracted from 461 patients of the randomly selected prospective Kardiovize study based on Brno, Czech Republic. Lipids species were measured by a hyphenated mass spectrometry technique, and were associated with poor CVH scores, as defined by the American Heart Association. Phosphatidylcholine (PC), phosphatidylethanolamine (PE), lysophosphatidylcholine (LPC), lysophosphatidylethanolamine (LPE) species were significantly lower in ideal and intermediate scores of health dietary metric, blood pressure, total cholesterol and blood fasting glucose compared to poor scores. Current smokers presented higher levels of PC, PE and LPE individual species compared to non-smokers. Ceramide (Cer) d18:1/14:0 was altered in poor blood pressure, total cholesterol and fasting blood glucose metrics. Poor cardiovascular health metric is associated with a specific phospho- and sphingolipid pattern. Circulatory lipid profiling is a potential biomarker to refine cardiovascular health status in primary prevention strategies.
\end{abstract}

Keywords: cardiovascular health; lipidomics; sphingolipids; phospholipids; mass spectrometry

\section{Introduction}

Cardiovascular diseases (CVD) represent the most common cause of mortality and morbidity among adults with roughly $30 \%$ of deaths worldwide [1]. The most important behavioral risk factors of CVD are unhealthy diet, physical inactivity, and tobacco use. The effects of these behavioral risk factors may cause hypertension, dysglycemia, dyslipidemia and overweight/obesity. In 2010, the AHA suggested an ideal cardiovascular health $(\mathrm{CVH})$ score to improve the cardiovascular health of the American population by $20 \%$ and to reduce deaths from CVD and stroke by $20 \%$ in 2020 [2]. The ideal CVH score reports the presence of seven ideal health parameters (non-smoking, body mass index $<25 \mathrm{~kg} / \mathrm{m}^{2}$, moderate and/or vigorous physical activity, a healthy diet consistent with current guideline recommendations, untreated total cholesterol $<5.17 \mathrm{mmol} / \mathrm{L}$, untreated fasting blood glucose $<5.55 \mathrm{mmol} / \mathrm{L}$ and untreated blood pressure $<120 /<80 \mathrm{~mm} \mathrm{Hg}$ ) [2] The Kardiovize study is a prospective cohort study to investigate the complex relationship of CVD and outcomes with a range of biological, psychosocial, environmental, behavioral, and economic factors in the urban population of Brno, the 2nd largest city (after the capital 
city, Prague) of Czech Republic, a landlocked country located in the heart of Europe [3-13]. The Kardiovize study evaluated $\sim 1 \%$ of the population of Brno and revealed that only $19 \%$ of the people aged 25-64 years old have an ideal CVH score [14]. In addition to traditional lipid risk factors [low density lipoproteins (LDL) and very low density lipoproteins (LDL) cholesterol, triglycerides], alterations in the composition of other circulatory lipid classes-such as sphingolipids and phospholipids-may contribute to the pathogenesis of CVD, as a result of dietary habits, environmental factors or genetic background. Phospholipids and sphingolipids are mainly found in the cell membrane, and the main structural difference is that phospholipids consist of a glycerol backbone whereas sphingolipids consist of a sphingosine backbone. Abnormal metabolism of these lipid species has been related to inflammation, oxidative stress, endothelial erosion and atherosclerotic CVD [15-17]. Circulating lipids, including sphingolipids and phospholipids have been proposed to participate to the development of CVD: the trajectory of lipidomic abnormalities has been followed longitudinally in healthy subjects, or in patients who suffered minor or major cardiovascular events [18-20]. A complex relationship among individual sphingolipid and phospholipid species, anthropometric and metabolic parameters has been reported in an Australian cohort of 640 subjects with prediabetes [21]. In a Canadian cohort of 990 teenagers, few glycerophosphocholine (GPC) metabolites were associated with blood pressure, visceral fat, and fasting insulin [22]. Inclusion of plasma lipidomic profiles has been recently reported to improve upon traditional risk factors for the prediction of cardiovascular events in patients with diabetes enrolled in the American ADVANCE trial and the Italian Bruneck study $[18,23]$. Cardiovascular primary prevention is crucial for risk stratification and to prevent the insurgence of CVD, in subjects that did not suffer yet of CVD or of cardiovascular events [24,25]. There are no population-based cohort studies evaluating whether circulating phospholipid and sphingolipid profiles can discriminate between poor and intermediate/ideal CVH, in absence of CVD, as defined by the AHA [2]. In addition, there are no studies involving lipidomic analysis in Central Europe, where CVD is extremely prevalent, designed to prevent CVD. The objective of the present study is to determine the sphingo- and phospho-lipid profile [ceramide (Cer), sphingomyelin (SM), phosphatidylcholine (PC), phosphatidylethanolamine (PE), lysophosphatidylcholine (LPC), lysophosphatidylethanolamine (LPE)] using Liquid Chromatography Electrospray Ionization Tandem Mass Spectrometric (LC-ESI/MS) in the Kardiovize study associated with the cardiovascular health score and individual health parameters (BMI, smoking status, health score diet, physical activity, blood pressure, fasting blood glucose and cholesterol levels). The aim of this study was to identify lipid species that could outperform as biomarkers for cardiometabolic risk stratification in subjects without CVD.

\section{Results}

\subsection{Study Population}

The mean age was $54.3 \pm 17.4$ years old and $56.1 \%$ were men. The distribution of $\mathrm{CVH}$ metrics is reported in Table S1. From the 461 subjects analyzed, the highest distribution corresponds to the ideal fasting blood glucose score with $78.7 \%$ of subjects, followed by the ideal smoking status (54.7\%). The ideal and intermediate scores are around $40 \%$ for each score in physical activity, body mass index (BMI), blood pressure, and total cholesterol metrics. The healthy dietary metric is composed of intermediate scores $(96.7 \%)$ and poor scored (3.3\%). Overall, 203 participants reported poor CVH, 238 intermediate $\mathrm{CVH}$, and only 20 participants ideal CVH. In this study population, approximately 108 individual lipid species were thus identified including 32 PC species, 23 PE species, 10 SM species, 17 LPC species, 15 LPE species, and 11 Cer species.

\subsection{Comparison of Lipid Species across Categories of Behavioral CVH Metrics}

Behavioral CVH metrics are composed of smoking, dietary metric, BMI, and physical activity, which were associated with each lipid class and species. 
First lipid species across categories of behavioral CVH metrics were compared. In the smoking metric, 6 lipid species were selected at the level of FDR $<0.05$ which varied between participants with poor, intermediate, or ideal CVH (Figure S1 and Table S2). Never and former smokers exhibited higher levels of LPE 22:6, LPE 16:0, LPE 20:5, PC 14:1_14:1, PE 18:2_16:1 and PE 18:0_18:0 than current smokers (Figure 1).

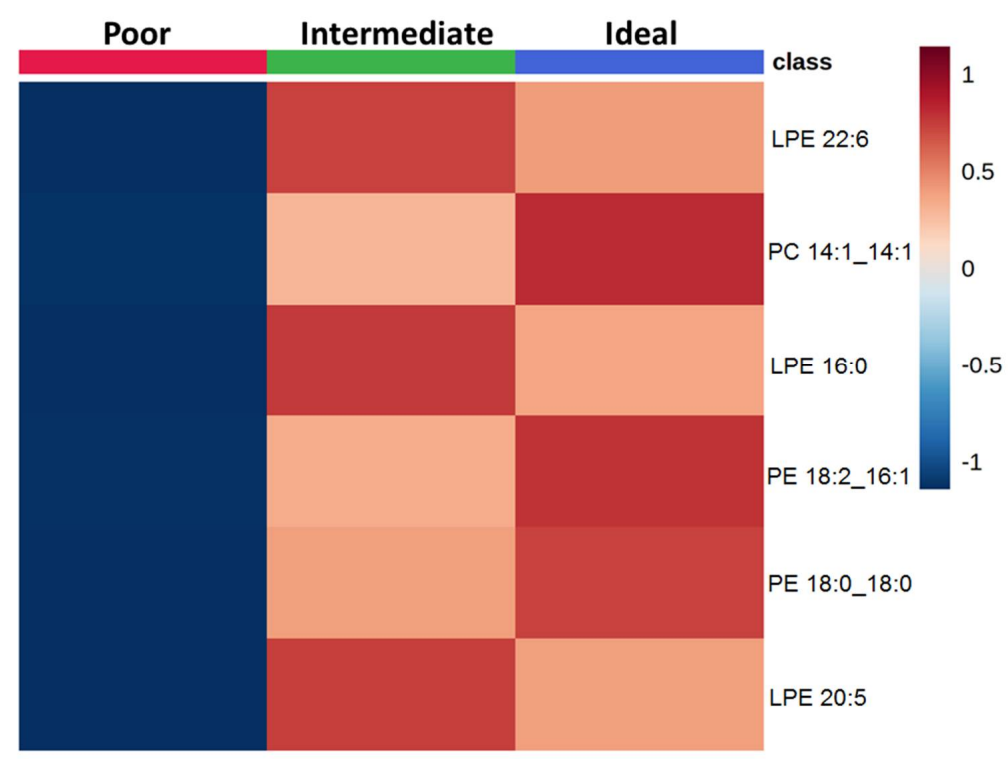

Figure 1. Comparison of lipid species across categories of smoking status. The heatmap shows the average concentrations of important lipid features identified by the ANOVA analysis and grouped by categories of smoking status.

The comparison of lipid species between participants with poor or intermediate level of the health diet metric was reported in Figure 2. No participants reported an ideal healthy diet score. Participants with intermediate health diet score exhibited significantly lower levels of PC 20:0_20:1, PC 20:0_20:3, PE 18:0_18:2, LPE 22:6, LPE 18:0, PE 16:0_20:2, LPE 16:0, PC 18:0/18:0, PE 16:0_20:3, LPE 20:5, PE 18:2_20:2, PC 16:0_18:0, PE 18:1_22:6, PE 16:0_18:2, PC 16:0_20:2, LPC 20:1, LPE 18:2, and PC 18:0_20:0 than those with poor score (FCs are reported in Table 1). No significant difference in lipid concentrations was evident across categories of physical activity and BMI (Figures S2 and S3).

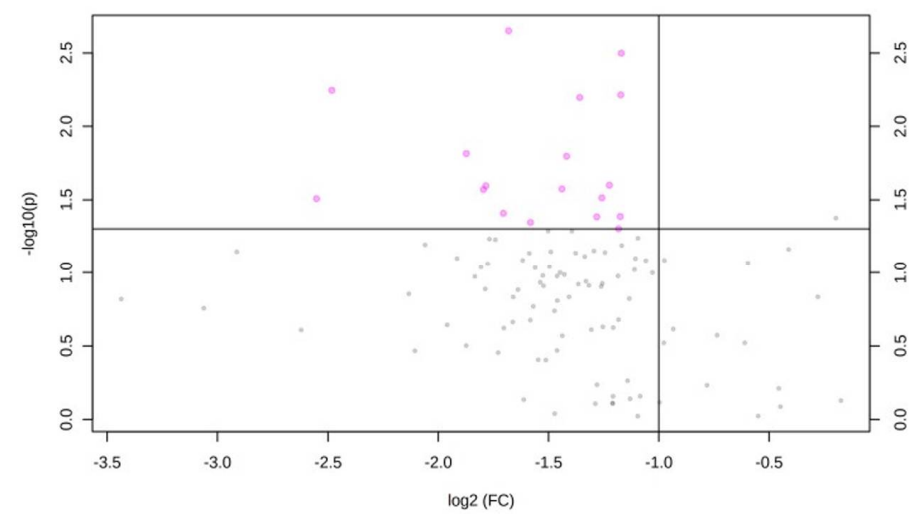

Figure 2. Comparison of lipid species across categories of diet. Volcano plot of important lipid features identified by the comparison between poor and intermediate dietary categories. This plot shows the magnitude (log 2 Fold-change of intermediate versus poor category; $x$-axis) and significance ( $-\log 10 p$-value; $y$-axis) for the comparison of lipids between intermediate and poor scores of dietary $\mathrm{CVH}$ metric. Lipid species with fold-change $>2$ or $<0.5$ and $p$-value $<0.05$ are represented as pink dots. 
Table 1. Important lipid features selected, fold changes (FCs) and $p$-values.

\begin{tabular}{ccc}
\hline Lipid Species & Fold-Change & $p$-Value \\
\hline PC 20.0/20.1 & 0.31 & 0.002 \\
PC 20.0/20.3 & 0.45 & 0.003 \\
PE 18.0/18.2 & 0.18 & 0.006 \\
LPE 22.6 & 0.44 & 0.006 \\
LPE 18.0 & 0.39 & 0.006 \\
PE 16.0/20.2 & 0.27 & 0.015 \\
LPE 16.0 & 0.37 & 0.016 \\
PC 18.0/18.0 & 0.43 & 0.025 \\
PE 16.0/20.3 & 0.29 & 0.025 \\
LPE 20.5 & 0.37 & 0.027 \\
PE 18.2/20.2 & 0.29 & 0.027 \\
PC 16.0/18.0 & 0.42 & 0.031 \\
PE 18.1/22.6 & 0.17 & 0.031 \\
PE 16.0/18.2 & 0.31 & 0.039 \\
PC 160/202 & 0.44 & 0.041 \\
LPC 20.1 & 0.42 & 0.041 \\
LPE 18.2 & 0.33 & 0.045 \\
PC 18.0/20.0 & 0.44 & 0.049 \\
\hline
\end{tabular}

\subsection{Comparison of Lipid Species across Categories of Clinical CVH Metrics}

Lipid concentrations across categories of clinical CVH metrics (blood pressure, total cholesterol, and blood fasting glucose) were compared. In general, the ideal level for clinical $\mathrm{CVH}$ metrics was related to lower lipid concentrations. Concerning the blood pressure metric, 53 lipid species were identified that significantly varied across categories (Figure S4 and Table S3), with a decreasing gradient from poor to ideal level (Figure 3A). Similarly, 5 lipid species significantly differed across categories of the total cholesterol metric (Figure S5 and Table S4). Specifically, PC 18:0_20:5, PE 18:0_20:5, PC 16:0_20:5 and PE 18:1_22:6 decreased from poor to ideal level, while Cer d18:1/14:0 increased (Figure 3B). The increased concentration of Cer d18:1/14:0 was also observed in participants with blood fasting glucose metrics at the ideal level, while other 18 lipid species were under-represented when compared with participants at poor level (Figures 3C and S6 and Table S5).

\subsection{Effect of CVH Status on Lipid Profiles}

Lipid concentrations were associated with participants with poor, intermediate, or ideal CVH status. Based on the ANOVA analysis 59 lipid species were selected with False Discovery Rate (FDR) $<0.05$ that varied across the categories (Figure S7 and Table S6). As reported for clinical CVH metrics, a decreasing trend of all lipid species from poor to ideal CVH status (Figure 4) was observed.

In the PLS-DA, using the CVH status (poor, intermediate, and ideal) as the response variable and lipid species as predictor variables. PLS-DA is a popular supervised algorithm for clustering or classification problems that aims to optimize separation between different groups of samples and to select discriminative variables. Accordingly, the score plot reported in Figure 5A showed that the two PLS-DA components explained $45.6 \%$ and 5.5\% of the variability, respectively. In this plot, a partial separation of participants with poor, intermediate, and ideal CVH status was observed, which was confirmed by the permutation test (Figure S8). The VIP analysis also indicated the most important lipid species involved in this discrimination, including PE 18:0_20:5, PE 18:0_22:4, PE 16:0_16:1, PC 18:0_20:5, PC 16:0_20:5, PE 16:0_20:3, PC 16:0_16:1, LPE 20:3, SM d18:1/18:1, PC 18:0_20:3, PC 18:1_20:5, PE 18:1_22:4, PE 18:1_22:6, and PE 16:0_18:2 (Figure 5B). 


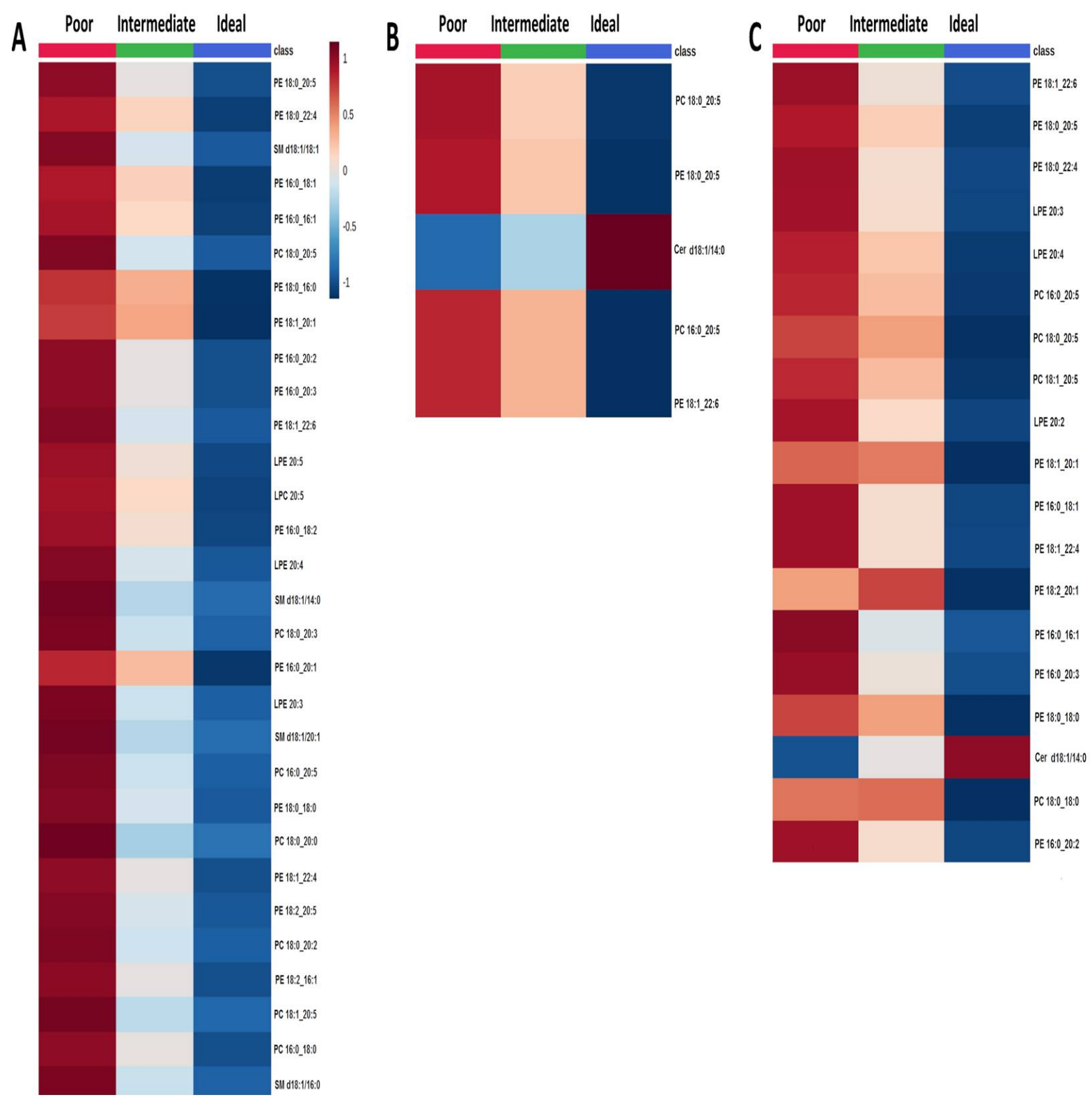

Figure 3. Comparison of lipid species across categories of 564 (A) blood pressure, (B) total cholesterol, and (C) fasting blood glucose metrics. The heatmap shows the average concentrations of important lipid features identified by the ANOVA analysis and grouped by categories of each metric.

The top-three lipid species (i.e., those with VIP >1.7) across categories of CVH status were compared. Concentrations of PE 16:0_16:1, PE 18:0_20:5 and PE 18:0_22:4 decreased from poor to ideal CVH status (Figure 6). 


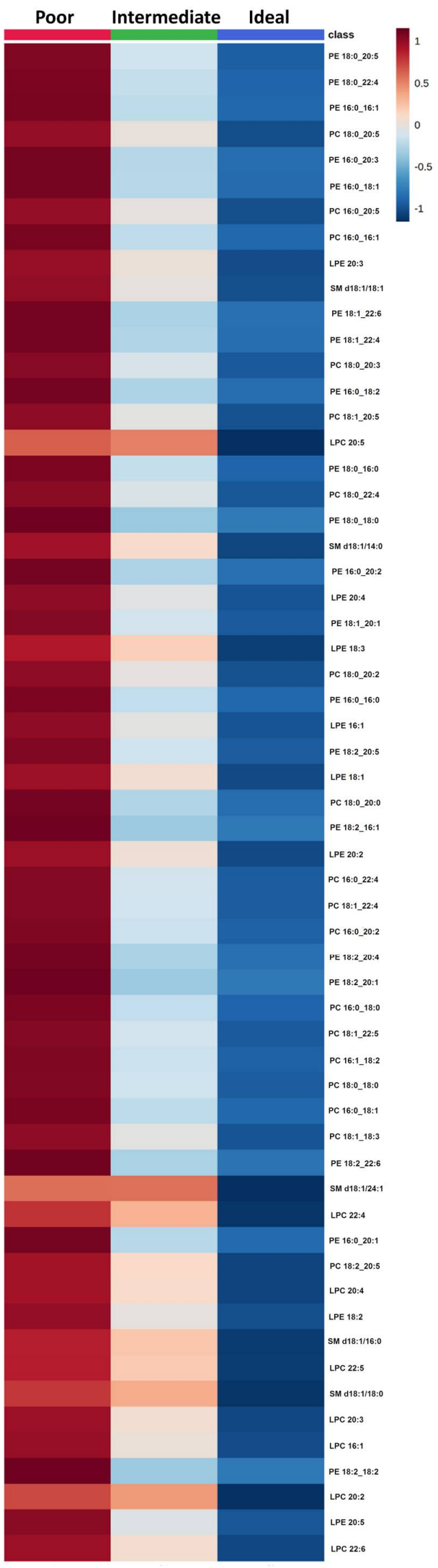

Figure 4. Comparison of lipid species across categories of cardiovascular health. The heatmap shows the average concentrations of important lipid features identified by the ANOVA analysis and grouped by categories of cardiovascular health. 
A

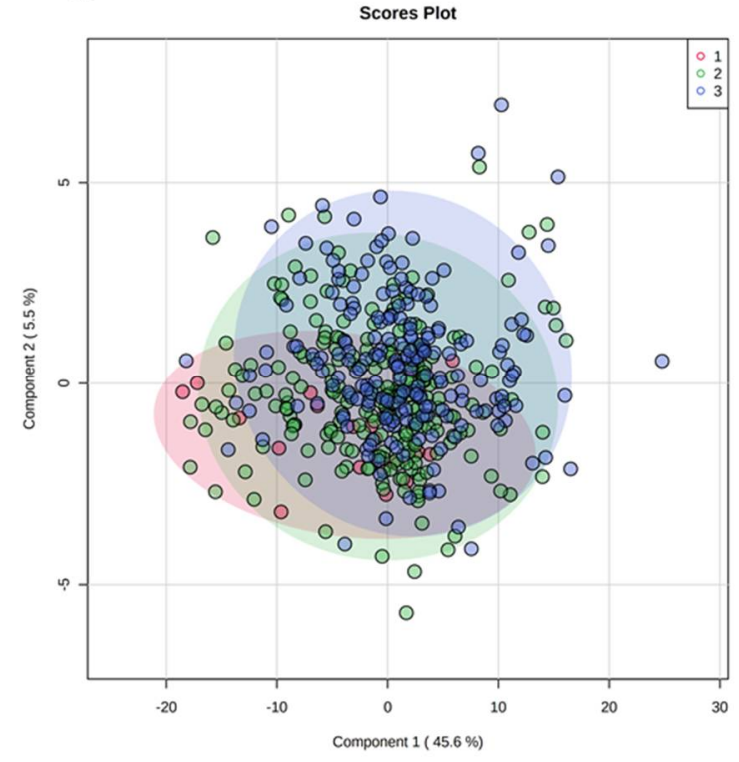

B

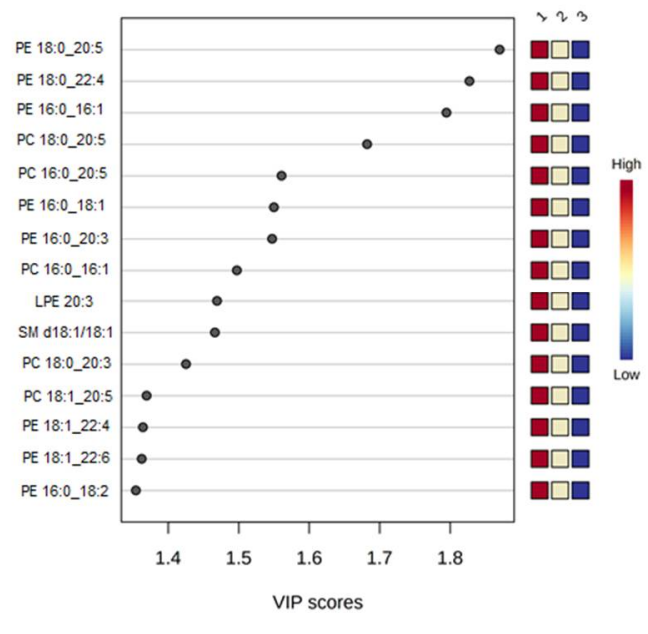

Figure 5. Outputs of the PLS-DA of lipid species on the cardiovascular health status. (A) PLS-DA scoreplot and (B) importance of variables ranked by Variable Importance in Projection (VIP) score for the comparison between categories of cardiovascular health. 1 = Poor; 2 = intermediate; 3 = ideal.

A

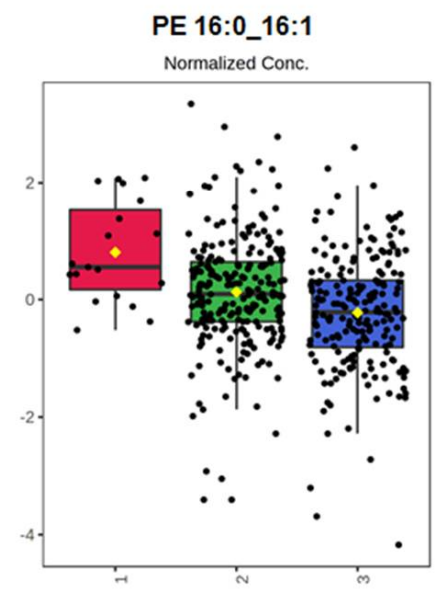

B

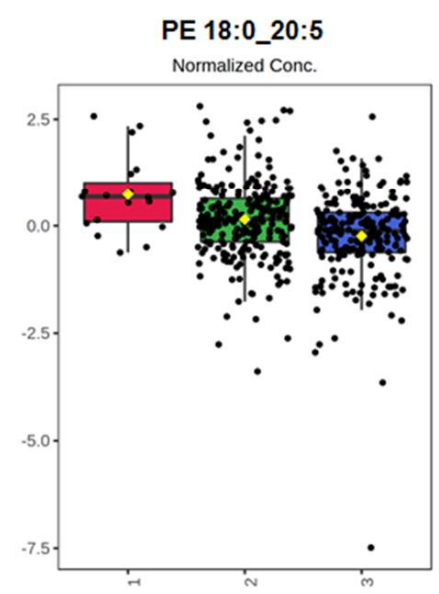

C

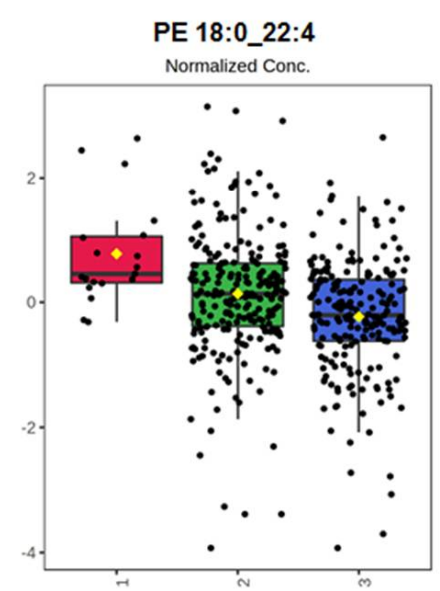

Figure 6. The top-three discriminant lipid species (A) PE 16:0_16:1, (B) PE 18:0_20:5, and (C) PE 18:0_22:4 across categories of $\mathrm{CVH}$ status. The boxplots show lipid species with VIP $>1.7$.

\section{Discussion}

In this study, the cardiovascular health parameters from an ideal to a poor score were associated with different concentrations of circulatory sphingolipids and phospholipids. Concretely, two main patterns were observed: (1) lower levels of PC, PE, LPC, and LPE individual species were found in ideal and intermediate scores of health dietary metric (for blood pressure, total cholesterol, and blood fasting glucose levels), compared to poor scores, and (2) on the contrary, higher levels of distinct PC, PE, and LPE individual species were found in never or former smokers compared to current smokers. Sphingolipids and phospholipids associated with behavioral CVH metrics. PC, PE, LPC, and LPE are the four lipid classes associated with smoking and dietary health CVH scores. Smoking behavior is associated mainly with lower levels of PE and LPE lipid species when compared to never smokers or quitters. Weir et al. evaluated non-smokers and smokers in a Mexican-American cohort: PE species (PE 32:1, PE 34:1, PE 34:3, PE 36:1, PE 36:4 and PE 36:5) and LPE species (LPE 18:1 and LPE 18:2) among others lipid classes were 
associated to smoking status [26]. A statistically significant correlation between smoking and increased total serum cholesterol, LDL and VLDL cholesterol, and triglyceride serum concentrations has been reported; this in turn participates to an increase of pro-atherogenic lipid oxidation $[27,28]$. Daily fat dietary intake contributes to modify the lipid profile. Plasma phospholipid fatty acids indicate the intake of fatty acids in the past few days [29]. Plasma saturated fat content is associated with dietary saturated fat but can be identified endogenously [30]; dietary intake is the main source of Omega-6 and Omega-3 PUFA [30]. In this study, lower levels of saturated lipid species (PC 18:0_18:0, PC 18:0_20:0, LPE 18:0, LPE 16:0), PC and PE, (PC 20:0_20:1, PE16:0_20:2, PC20:0_20:3) were found in participants with intermediate dietary health scores. High levels with species containing PUFA should be found in intermediary dietary scores that could indicate the presence of omega- 3 and omega-6 lipid species consumed in the diet. However, ideal dietary health scores were not present in this population that could confirm this hypothesis. Sphingolipids and phospholipids associated with clinical CVH metrics. The blood pressure metric is associated with a higher number of individual lipids and classes than other clinical or behavioral scores. Fourteen PC species, 18 PE species, 2 LPC species, 8 LPE species, 10 SM species were found more abundant in poor blood pressure scores when compared to ideal blood pressure scores. In particular, the blood pressure metric was the only cardiovascular parameter associated with sphingomyelin species (SM d18:1/18:1, SM d18:1/14:0, SM d18:1/20:1, SM d18:1/16:0, SM d18:1/18:0, SM d18:1/20:0, SM d18:1/22:1, SM d18:1/24:1, SM d18:1/26:1, SM d18:1/24:0). The present findings are consistent with the literature. Shahin et al. reported that patients with hypertension consuming hydrochlorothiazide anti-hypertensive drug exhibited alterations of the sphingolipids including 4 SMs (N24:2, N24:3, N16:1, and N22:1) [31]; while Zheng et al. observed that higher SM levels were associated with a pattern of non-dipper hypertension [32]. Fernandez et al. also reported that SM38:2 was the only SM molecular species related to future cardiovascular events [33]. Sphingolipids, including ceramides and sphingosine 1-phosphate, have been associated with blood pressure homeostasis and vascular functions but not sphingomyelin [32]. However, HDL-sphingomyelin decreases after remarkable weight loss was associated with blood pressure improvement [33]. On the other hand, dyslipidemia linked to poor fasting blood glucose score is likely related to prediabetic or diabetic state, as demonstrated by recent lipidomic studies analyzing the lipidomic alterations in these patients [21,34,35]. In this present study, PE individual species are the main lipids modified within the poor fasting blood glucose metric category. Other studies have also found PE positively associated with the development of diabetes $[35,36]$, corroborating the current findings. In general, high levels of total cholesterol are related to elevated LDL-cholesterol and apolipoprotein-B containing lipoproteins that transport cholesterol and other lipids throughout the body and initiate atherosclerosis [37]. Alterations of circulatory lipids in both poor fasting blood glucose and poor total cholesterol metric might be due to an increase in lipoprotein production and dysregulation of PC and PE containing omega-3 and omega-6 PUFA, which in turn are crucial for the synthesis of eicosanoids [34,38]. Moreover, adequate levels of lipid species containing PUFA and their biologically active derivatives have been shown to favor synaptic function and to prevent brain inflammation, providing a link between cardiometabolic and brain health [39]. Sphingolipids and phospholipids associated with $\mathrm{CVH}$ total scores. Across all categories of CVH metrics, PE species (PE 16:0_16:1, PE 18:0_20:5, and PE 18:0_22:4) are the most affected, decreasing significantly in poor versus ideal CVH status. A recent animal study reported that alterations in plasma lipid species might reveal metabolic health; plasma lipids are impacted by heritable factors despite dietary intake, and lipid species are modulated by loci spread across the genome [40]. In primary prevention, this study provides a lipidomic signature that can be used as a surrogate for cardiovascular health in absence of CVD events. Sphingolipid and phospholipid biosynthetic pathways associated to CVH. Among phospholipids, PC, LPC, PE, LPE, and sphingolipids (SM and Cer) play a major role in the pathogenesis of CVD [17,41]. Figure 7 indicates the lipid pathway of the lipids analysed in this present study. 


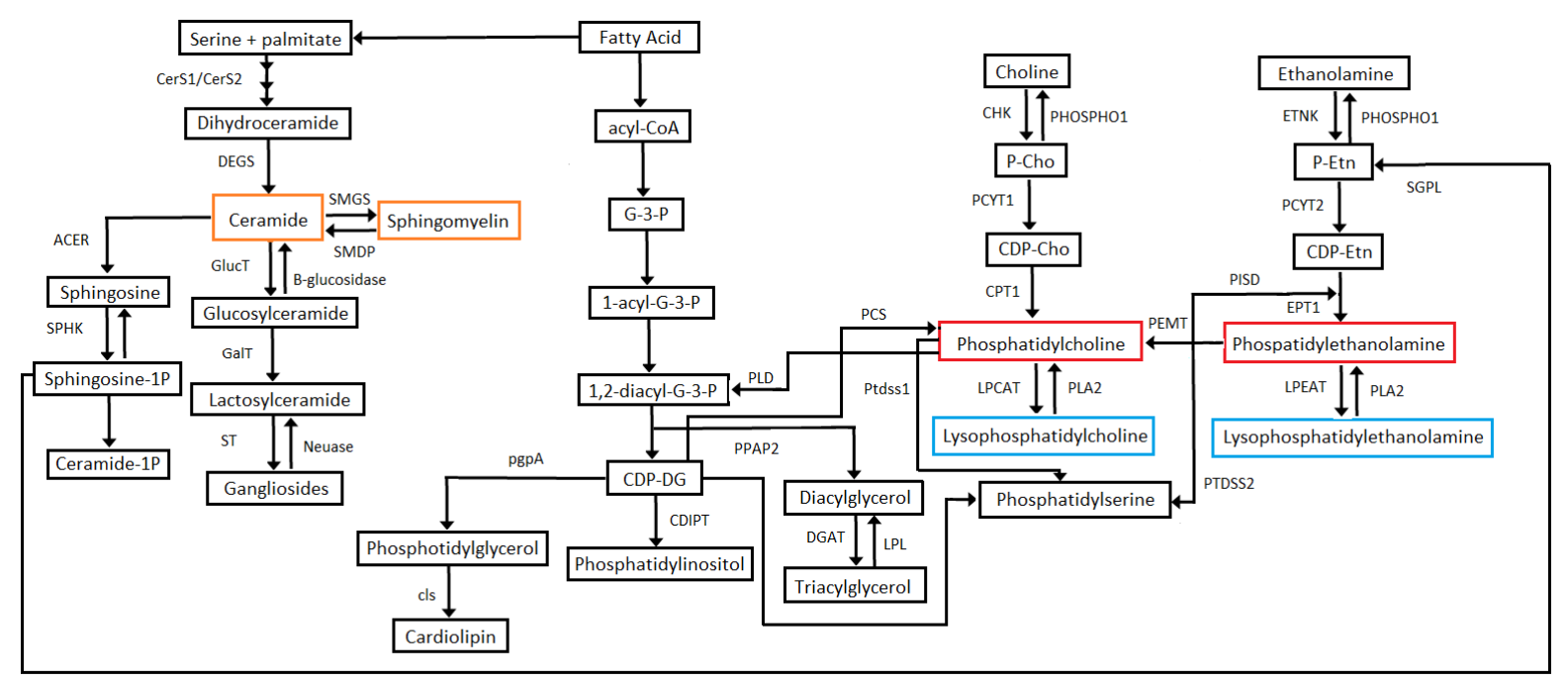

Figure 7. Schematic Representation of lipid metabolic pathways. Metabolite abbreviations: G-3-P, sn-Glycerol 3Phosphate; 1-acyl-G-3-P, 1-Acyl-sn-glycerol 3-phosphate; 1,2-diacyl-G-3-P, 1,2-Diacyl-sn-glycerol 3-phosphate; CDP-DG, CDP-1,2-diacylglycerol; P-Cho, Choline Phosphate; CDP-Cho, CDP-choline; P-Etn, Phosphoethanolamine; CDP-Etn, CDP-ethanolamine. Enzyme abbreviations: CerS1/CerS2, Ceramide Synthase 1/Ceramide Synthase 2; DEGS, sphingolipid delta-4 desaturase; SMGS, sphingomyelin synthase; SMDP, Sphingomyelin Phosphodiesterase; ACER, alkaline ceramidase; SPHK, sphingosine kinase; GlucT, Glucosylceramide-Transferase; GalT, Galactosylceramide-Transferase; ST, Sialyl-Transferase; Neusase, Neuraminase; pgpA, phosphatidylglycerophosphatase A; cls, cardiolipin synthase; PLD, Phospholipase D1/2; PPAP2, phosphatidate phosphatase; CDIPT, CDP-diacylglycerol-inositol 3-phosphatidyltransferase; CHK, choline kinase; PHOSPHO1, phosphoethanolamine/phosphocholine 586 phosphatase; PCYT1, choline-phosphate cytidylyltransferase; CPT1, diacylglycerol cholinephosphotransferase; PCS, phosphatidylcholine synthase; DGAT, diacylglycerol O-acyltransferase; LPC, lipoprotein lipase; Ptdss1, phosphatidylserine synthase 1; LPCAT, lysophosphatidylcholine 650 acyltransferase; PLA2, secretory phospholipase A2; ETNK, Ethanolamine Kinase; PCYT2, ethanolaminephosphate cytidylyltransferase; PISD, phosphatidylserine decarboxylase; EPT1, ethanolaminephosphotransferase; PEMT, phosphatidylethanolamine N-methyltransferase; LPEAT, lysophospholipid acyltransferase; PTDSS2, phosphatidylserine synthase 2. Lipid species analysed in the present study: Orange rectangles = sphingolipids, red rectangles $=$ phospholipids, blue rectangles $=$ lysophospholipids.

PC, PE, LPC, LPE are structural and functional cell membrane components [42]. Approximately $90 \%$ of cardiac PC phospholipid is synthesized by the Cytidine Diphosphate (CDP)-choline pathway $[43,44]$. PE is synthesized by the CDP-ethanolamine pathway and the phosphatidylserine decarboxylase pathway. PE in turn is converted to PC by phosphatidylethanolamine N-methyltransferase (PEMT) [42]. PC biosynthesis is tightly associated with plasma lipoprotein homeostasis [45]. It is well known that PC in lipoproteins is converted to triglycerides in the liver [46,47]. Lysophospholipids (LPC and LPE) are deacylated products of phospholipids with one fatty acid chain and are produced by phospholipase A (PLA1 or PLA2) [48]. Production of LPC also results from lecithin-cholesterol acyltransferase activity or hepatic secretion [49]. Ceramides are complex sphingolipids biosynthesized in all tissues from saturated fat and sphingosine [50]. SM is the most abundant sphingolipid in lipoproteins and total plasma after PC [51]. The sphingomyelinceramide pathway has also been involved in the development of atherosclerosis since this pathway has a role in the oxidized LDL-induced of smooth muscle cells [52]. Interestingly, links between SM and ceramide levels were not identified. The ceramide d18:1/14:0 was the only ceramide species affected in this study. Elevated levels of circulating ceramides and ceramide ratios have been identified as risk factors for major cardiovascular events [53]. Nevertheless, the ceramide composed of d18:1/14:0 showed an opposite effect with higher values in ideal blood pressure, cholesterol, and glucose CVH scores. There are only a few studies indicating evidence of plasma Cer d18:1/14:0 as a risk or protective factor for CVD. For instance, Yao et al. reported that Cer d18:1/14:0 is a biomarker to identify the risk 
of acute coronary syndrome [54]. More studies are needed to understand the role of Cer d18:1/14:0. Comparisons with other studies and what does the current work add to the existing knowledge. Previous studies analysed the association between lipid species and CVD [19,55,56], or between lipid species and single metabolic risk factors [40,57]. This is the first study providing a sphingolipidomic and phospholipidomic signature that can be used as a fast surrogate for systemic cardiovascular health in absence of CVD events, currently requiring multiple biochemical and anthropometric assessments, if validated in independent cardiovascular cohorts. LC-ESI/MS platforms have become a widespread technology within clinical reference and referral laboratories world-wide, penetrating into hospitals and regional clinical laboratories. Therefore, a strength of this study relies a robust LC-ESI/MS platform to define in depth sphingo- and phospho- lipid profiles, combined with unique multidimensional cardiovascular health data, in a large size population-based cohort in Central Europe, a region where CVD incidence is the highest in the world. Limitations of the study include: (1) LC/MS method used provides information of the carbon chain length and number of bonds, but does not provide information of the position of the double bonds; (2) other phospholipids and plasmalogens were not quantified in this study, and triglycerides and diacylglycerides are species not retained in the column used; (3) participants with ideal health dietary metrics to cover all optimal categories were not found. The stringency of AHA criteria for $\mathrm{CVH}$ deserves careful consideration [58]; it is not possible from cross-sectional analyses to establish a causal link between altered lipidomic profiles and $\mathrm{CVH}$.

\section{Materials and Methods}

\subsection{Study Population and Data Collection}

The Kardiovize study is a multidisciplinary cross-sectional epidemiological project [3-14], including 2160 participants aged $25-64$, and 270 subjects aged $\geq 65$. 65 years was thus used as cut-off point to define older adults. For this lipidomic analysis, 259 participants aged 25 to 64 years old (young cohort) were randomly selected, while 212 participants aged 65 to 89 years old that were selected to participate in this study were parents or foster parents. Therefore, older participants had a family relationship with younger participants. Ten participants aged 65 to 89 years old who presented a history of cardiac major events, such as stroke and coronary events, were excluded from this analysis. The present study includes 461 female and male participants aged 25-89 years old. Participants signed the informed consent and completed a health interview questionnaire, smoking history, medication intake, physical activity - using a validated Czech translation of the international physical activity questionnaire (IPAQL)—and a food frequency questionnaire (FFQ). Participants underwent a physical examination assessing blood pressure measurement using an automated office measurement device (BpTRU, model BPM 200; BpTRU Medical Devices Ltd., Vancouver, BC, Canada). Height and weight were measured using a digital medical scale with meter (SECA 799; SECA, GmbH and Co. KG, Hamburg, Germany). Laboratory tests were performed with 12-h fasting full blood samples using a Modular SWA P800 analyser (Roche, Basel, Switzerland) or enzymatic colorimetric methods (RocheDiagnostics $\mathrm{GmbH}$, Hamburg, Germany), all assessed in St Anne's University Hospital by trained research nurses. Serum blood samples were stored at $-80^{\circ} \mathrm{C}$ until lipidomic analysis was performed [59]. The study protocol was approved by the ethics committee of St Anne's University Hospital, Brno, Czech Republic (reference 2 G/2012), in accordance with the Declaration of Helsinki.

\subsection{Lipid Extraction and LC-ESI/MS Analysis}

Serum lipids were extracted [60]. Briefly, $100 \mu \mathrm{L}$ of serum containing deuterated lipid internal standard (Equisplash, Avanti Polar Lipids, Alabaster, AL, USA) was mixed with chloroform/methanol $(2: 1, v / v)$ and $\mathrm{CaCl} 2(0.025 \%, w / v)$ and shake with a thermoshaker (TS-100, Biosan, Riga, Latvia) at $1300 \mathrm{rpm}$. Samples were sonicated for $30 \mathrm{~s}$ (Kraintek 18, Hradec Kralove, Czech Republic). After centrifugation $\left(16,000 \times g, 15 \mathrm{~min}, 4{ }^{\circ} \mathrm{C}\right.$; 
Eppendorf 5427R), the lower phase was extracted and the top layer was processed again following the same Folch extraction. Layers were combined and were evaporated using a speed vacuum. Lipid extracts were reconstituted with acetonitrile:water $(3: 1, v / v)$. Lipid extractions were analyzed using a HPLC system composed of a Thermo Scientific Dionex UltiMate ${ }^{\mathrm{TM}} 3000$ RSLCnano system connected to a ABSciex QTRAP 6500 system, as previously described [61]. A Waters Acquity UPLC BEH HILIC, $1.7 \mu \mathrm{m}, 2.1 \times 100 \mathrm{~mm}$ (Waters, Milford, MA, USA) column with a guard column, Acquity UPLC BEH HILIC $1.7 \mu \mathrm{m}$ VanGuard Pre-Column $2.1 \times 5 \mathrm{~mm}$ (Waters, Milford, MA, USA). The column was eluted a flow rate of $400 \mu \mathrm{L} / \mathrm{min}$ a linear gradient from 0 to $10 \mathrm{~min}$ to $80 \%$ mobile phase $\mathrm{A}$ (water/acetonitrile [5:95, $v / v]$ with $10 \mathrm{mM}$ ammonium acetate) and over $11 \mathrm{~min}$ to $98 \%$ mobile phase B (water/acetonitrile [50:50, v/v] with $10 \mathrm{mM}$ ammonium acetate; $\mathrm{pH}=8.2$. . Mass spectrometry analyses were performed using electrospray ionization in the positive/negative mode and MRM scan, with a resolution of $0.7+/-0.1 \mathrm{u}$ [Full Width at Half Maximum (FWHM)]. MS additional parameters were as follows: curtain gas (CUR) 35; temperature (TEM) $500{ }^{\circ} \mathrm{C}$; Ion Source Gas 1 (GS1) 40; Ion Source Gas 2 (GS2) 50; Ion spray voltage 5200/-4500. A library of theoretical precursor ions was generated for Cer, SM, PC, PE, LPE, and LPC varying the length of ceramide or fatty acid carbon chain. The characterization of phospholipid and sphingolipid classes and individual species was achieved in positive mode (Cer and SM) with the formation of $[\mathrm{M}+\mathrm{H}]^{+}$, in negative mode (PE, LPE) with the formation of $[\mathrm{M}-\mathrm{H}]^{-}$and $[\mathrm{M}+\mathrm{OAc}]^{-}$for LPC and PC. The collision energy varies from 43 to $51 \mathrm{eV}$. Data analysis was carried out using the Skyline daily 4.2.1.19058 software (MacCoss Lab, Department of Genome Sciences, University of Washington, Seattle, WA, USA).

\subsection{Statistical Analyses}

Lipidomic analyses were performed on the MetaboAnalyst web tool (www.metaboanalyst. $\mathrm{ca}$ (accessed on 27 October 2021)). Prior to further analysis, missing values were replaced using the k-nearest neighbors (KNN) algorithm, and data were normalized using their median, log-transformed e auto-scaled. The concentration of lipid species was compared for each $\mathrm{CVH}$ metric, and across categories of $\mathrm{CVH}$ status. The volcano plot-which combines fold-change analysis and $t$-test-was generated for two-group comparisons. Lipid features with fold-change threshold $>2$ or $<0.5$ and $p$-value $<0.05$ were considered statistically significant. The one-way Analysis of Variance (ANOVA) followed by Fisher's least significant difference (LSD) was applied for multigroup comparisons. Lipid features with adjusted $p$-value $<0.05$ using the False Discovery Rate (FDR) were considered statistically significant. In general, the average concentrations of important lipid features identified were compared and reported using a heatmap. The Partial Least Squares Discriminant Analysis (PLS-DA) was performed to predict the variability across categories of CVH status. The Variables Important in Projection (VIP) analysis was used to identify important lipid features that contributed to the separation between categories.

Supplementary Materials: The following are available online at https: / www.mdpi.com/article / 10.3390/metabo11110747/s1. Table S1: Distribution of CVH metrics among 461 Kardiovize participants, Table S2: Important lipid features identified by ANOVA analysis and Fisher's least significant difference method across CVH categories of smoking status, Table S3: Important lipid features identified by ANOVA analysis and Fisher's least significant difference method across CVH categories of blood pressure, Table S4: Important lipid features identified by ANOVA analysis and Fisher's least significant difference method across CVH categories of total cholesterol, Table S5: Important lipid features identified by ANOVA analysis and Fisher's least significant difference method across CVH categories of blood fasting glucose, Table S6: Important lipid features identified by ANOVA analysis and Fisher's least significant difference method across categories of CVH, Table S7: Definition of cardiovascular health metrics; Figure S1. ANOVA analysis of lipid species across CVH categories of smoking status. Lipid species with adjusted $p$-value (FDR) $<0.05$ are indicated in red., Figure S2: ANOVA analysis of lipid species across $\mathrm{CVH}$ categories of physical activity, Figure S3: ANOVA analysis of lipid species across CVH categories of BMI, Figure S4: ANOVA analysis of lipid species 
across CVH categories of blood pressure. Lipid species with adjusted $p$-value (FDR) $<0.05$ are indicated in red., Figure S5: ANOVA analysis of lipid species across CVH categories of total cholesterol. Lipid species with adjusted $p$-value (FDR) $<0.05$ are indicated in red., Figure S6: ANOVA analysis of lipid species across $\mathrm{CVH}$ categories of blood fasting glucose. Lipid species with adjusted $p$-value (FDR) $<0.05$ are indicated in red., Figure S7: ANOVA analysis of lipid species across categories of CVH. Lipid species with adjusted $p$-value (FDR) $<0.05$ are indicated in red., Figure S8: Classification performance and permutation test of the PLS-DA. (A) PLS-DA classification using different number of components. The red star indicates the best classifier. (B) PLS-DA model validation by permutation tests based on separation distance.

Author Contributions: I.M.R.S. performed proteomic/mass-spec analyses and interpreted the data; M.S. and J.R.M.-I. performed statistical analyses; I.M.R.S., G.B.S., J.P.G.-R., F.L.-J. and M.V. designed the work, drafted the manuscript and substantively revised it. All authors have read and agreed to the published version of the manuscript.

Funding: This work was supported by the National Program of Sustainability II (MEYS CR) (No. LQ1605), and by the European Regional Development Fund-Projects ENOCH (No. CZ.02.1.01/ 0.0/0.0/16_019/0000868) and MAGNET (No. CZ.02.1.01/0.0/0.0/15_003/0000492), and in part by the Mayo Clinic department of Cardiovascular Medicine and Marriot Heart Disease Research Program. The authors also thank the Support of mobility of researchers at FNUSA-ICRC CZ.02.2.69/ 0.0/0.0/16_027/0008359.

Institutional Review Board Statement: The study protocol was approved by the ethics committee of St Anne's University Hospital, Brno, Czech Republic (reference 2 G/2012), in accordance with the Declaration of Helsinki. All participants signed the informed consent and the consent for publication.

Informed Consent Statement: All participants signed the informed consent and the consent for publication.

Data Availability Statement: The datasets used and/or analysed during the current study are available from the corresponding author on reasonable request.

Acknowledgments: CIISB: Instruct-CZ Centre of Instruct-ERIC EU consortium, funded by MEYS CR infrastructure project LM2018127, is gratefully acknowledged for the financial support to the measurements at the CEITEC Proteomics Core Facility. The authors thank all the members of Kardiovize Brno 2030 team for their contributions in data acquisition, and they are indebted to Andrea Maugeri (University of Catania, Italy) for data analysis and graphic representation.

Conflicts of Interest: The authors declare no conflict of interest.

\section{References}

1. Roth, G.A.; Johnson, C.; Abajobir, A.; Abd-Allah, F.; Abera, S.F.; Abyu, G.; Ahmed, M.; Aksut, B.; Alam, T.; Alam, K.; et al. Global, Regional, and National Burden of Cardiovascular Diseases for 10 Causes, 1990 to 2015. J. Am. Coll. Cardiol. 2017, 70, 1-25. [CrossRef]

2. Lloyd-Jones, D.M.; Hong, Y.; Labarthe, D.; Mozaffarian, D.; Appel, L.J.; Van Horn, L.; Greenlund, K.; Daniels, S.; Nichol, G.; Tomaselli, G.F.; et al. Defining and setting national goals for cardiovascular health promotion and disease reduction: The American Heart Association's strategic Impact Goal through 2020 and beyond. Circulation 2010, 121, 586-613. [CrossRef]

3. Maugeri, A.; Hlinomaz, O.; Agodi, A.; Barchitta, M.; Kunzova, S.; Bauerova, H.; Sochor, O.; Medina-Inojosa, J.R.; Lopez-Jimenez, F.; Vinciguerra, M.; et al. Is Drinking Alcohol Really Linked to Cardiovascular Health? Evidence from the Kardiovize 2030 Project. Nutrients 2020, 12, 2848. [CrossRef]

4. Movsisyan, N.K.; Vinciguerra, M.; Lopez-Jimenez, F.; Kunzova, S.; Homolka, M.; Jaresova, J.; Cífková, R.; Sochor, O. Kardiovize Brno 2030, a prospective cardiovascular health study in Central Europe: Methods, baseline findings and future directions. Eur. J. Prev. Cardiol. 2018, 25, 54-64. [CrossRef]

5. Kunzova, S.; Maugeri, A.; Medina-Inojosa, J.; Lopez-Jimenez, F.; Vinciguerra, M.; Marques-Vidal, P. Determinants of Metabolic Health across Body Mass Index Categories in Central Europe: A Comparison Between Swiss and Czech Populations. Front. Public Health 2020, 8, 108. [CrossRef]

6. Maugeri, A.; Barchitta, M.; Kunzova, S.; Bauerova, H.; Agodi, A.; Vinciguerra, M. The association of social and behavioral factors with dietary risks in adults: Evidence from the Kardiovize Brno 2030 study. Nutr. Metab. Cardiovasc. Dis. 2020, 30, 896-906. [CrossRef]

7. Maugeri, A.; Hruskova, J.; Jakubik, J.; Hlinomaz, O.; Medina-Inojosa, J.R.; Barchitta, M.; Agodi, A.; Vinciguerra, M. How dietary patterns affect left ventricular structure, function and remodelling: Evidence from the Kardiovize Brno 2030 study. Sci. Rep. 2019, 9, 19154. [CrossRef] 
8. Maugeri, A.; Medina-Inojosa, J.R.; Kunzova, S.; Barchitta, M.; Agodi, A.; Vinciguerra, M.; Lopez-Jimenez, F. Dog Ownership and Cardiovascular Health: Results from the Kardiovize 2030 Project. Mayo Clin. Proc. Innov. Qual. Outcomes 2019, 3, $268-275$. [CrossRef]

9. Maugeri, A.; Hruskova, J.; Jakubik, J.; Kunzova, S.; Sochor, O.; Barchitta, M.; Agodi, A.; Bauerova, H.; Medina-Inojosa, J.R.; Vinciguerra, M. Dietary antioxidant intake decreases carotid intima media thickness in women but not in men: A cross-sectional assessment in the Kardiovize study. Free. Radic. Biol. Med. 2019, 131, 274-281. [CrossRef]

10. Maugeri, A.; Medina-Inojosa, J.R.; Kunzova, S.; Agodi, A.; Barchitta, M.; Sochor, O.; Lopez-Jimenez, F.; Geda, Y.E.; Vinciguerra, M. Sleep Duration and Excessive Daytime Sleepiness Are Associated with Obesity Independent of Diet and Physical Activity. Nutrients 2018, 10, 1219. [CrossRef]

11. Agodi, A.; Maugeri, A.; Kunzova, S.; Sochor, O.; Bauerova, H.; Kiacova, N.; Barchitta, M.; Vinciguerra, M. Association of Dietary Patterns with Metabolic Syndrome: Results from the Kardiovize Brno 2030 Study. Nutrients 2018, 10, 898. [CrossRef]

12. Hruskova, J.; Maugeri, A.; Podroužková, H.; Štípalová, T.; Jakubík, J.; Barchitta, M.; Medina-Inojosa, J.R.; Homolka, M.; Agodi, A.; Kunzova, S.; et al. Association of Cardiovascular Health with Epicardial Adipose Tissue and Intima Media Thickness: The Kardiovize Study. J. Clin. Med. 2018, 7, 113. [CrossRef]

13. Maugeri, A.; Kunzova, S.; Medina-Inojosa, J.R.; Agodi, A.; Barchitta, M.; Homolka, M.; Kiacova, N.; Bauerova, H.; Sochor, O.; Lopez-Jimenez, F.; et al. Association between eating time interval and frequency with ideal cardiovascular health: Results from a random sample Czech urban population. Nutr. Metab. Cardiovasc. Dis. 2018, 28, 847-855. [CrossRef]

14. Medina-Inojosa, J.R.; Vinciguerra, M.; Maugeri, A.; Kunzova, S.; Sochor, O.; Movsisyan, N.; Geda, Y.E.; Stokin, G.B.; LopezJimenez, F. Prevalence of ideal cardiovascular health in a Central European community: Results from the Kardiovize Brno 2030 Project. Eur. J. Prev. Cardiol. 2020, 27, 441-443. [CrossRef]

15. Zhong, S.; Li, L.; Shen, X.; Li, Q.; Xu, W.; Wang, X.; Tao, Y.; Yin, H. An update on lipid oxidation and inflammation in cardiovascular diseases. Free Radic. Biol. Med. 2019, 144, 266-278. [CrossRef]

16. Borodzicz, S.; Czarzasta, K.; Kuch, M.; Cudnoch-Jedrzejewska, A. Sphingolipids in cardiovascular diseases and metabolic disor-ders. Lipids Health Dis. 2015, 14, 55. [CrossRef]

17. Boffa, M.B.; Koschinsky, M. Oxidized phospholipids as a unifying theory for lipoprotein(a) and cardiovascular disease. Nat. Rev. Cardiol. 2019, 16, 305-318. [CrossRef]

18. Stegemann, C.; Pechlaner, R.; Willeit, P.; Langley, S.R.; Mangino, M.; Mayr, U.; Menni, C.; Moayyeri, A.; Santer, P.; Rungger, G.; et al. Lipidomics profiling and risk of cardiovascular disease in the prospective population-based Bruneck study. Circulation 2014, 129, 1821-1831. [CrossRef]

19. Mundra, P.; Barlow, C.K.; Nestel, P.J.; Barnes, E.H.; Kirby, A.; Thompson, P.; Sullivan, D.R.; Alshehry, Z.H.; Mellett, N.A.; Huynh, K.; et al. Large-scale plasma lipidomic profiling identifies lipids that predict cardiovascular events in secondary prevention. JCI Insight 2018, 3, 3. [CrossRef]

20. Vasile, V.C.; Meeusen, J.W.; Medina Inojosa, J.R.; Donato, L.J.; Scott, C.G.; Hyun, M.S.; Vinciguerra, M.; Rodeheffer, R.R.; LopezJimenez, F.; Jaffe, A.S. Ceramide Scores Predict Cardiovascular Risk in the Community. Arterioscler. Thromb. Vasc. Biol. 2021, 41, 1558-1569. [CrossRef]

21. Huynh, K.; Barlow, C.K.; Jayawardana, K.S.; Weir, J.M.; Mellett, N.A.; Cinel, M.; Magliano, D.; Shaw, J.E.; Drew, B.G.; Meikle, P.J. High-Throughput Plasma Lipidomics: Detailed Mapping of the Associations with Cardiometabolic Risk Factors. Cell Chem. Biol. 2019, 26, 71-84.e4. [CrossRef]

22. Syme, C.; Czajkowski, S.; Shin, J.; Abrahamowicz, M.; Leonard, G.; Perron, M.; Richer, L.; Veillette, S.; Gaudet, D.; Strug, L.; et al. Glyc-erophosphocholine Metabolites and Cardiovascular Disease Risk Factors in Adolescents: A Cohort Study. Circulation 2016, 134, 1629-1636. [CrossRef]

23. Alshehry, Z.H.; Mundra, P.A.; Barlow, C.K.; Mellett, N.A.; Wong, G.; McConville, M.J.; Simes, J.; Tonkin, A.M.; Sullivan, D.R.; Barnes, E.H.; et al. Plasma Lipidomic Profiles Improve on Traditional Risk Factors for the Prediction of Cardiovascular Events in Type 2 Dia-betes Mellitus. Circulation 2016, 134, 1637-1650. [CrossRef]

24. Visseren, F.L.J.; Mach, F.; Smulders, Y.M.; Carballo, D.; Koskinas, K.C.; Back, M.; Benetos, A.; Biffi, A.; Boavida, J.M.; Capodanno, D.; et al. 2021 ESC Guidelines on cardiovascular disease prevention in clinical practice. Eur. Heart J. 2021, 42, 3227-3337. [CrossRef]

25. Arnett, D.K.; Blumenthal, R.S.; Albert, M.A.; Buroker, A.B.; Goldberger, Z.D.; Hahn, E.J.; Himmelfarb, C.D.; Khera, A.; LloydJones, D.; McEvoy, J.W.; et al. 2019 ACC/AHA Guideline on the Primary Prevention of Cardiovascular Disease: A Report of the American College of Cardiology/American Heart Association Task Force on Clinical Practice Guidelines. Circulation 2019, 140, e596-e646. [CrossRef] [PubMed]

26. Weir, J.M.; Wong, G.; Barlow, C.; Greeve, M.A.; Kowalczyk, A.; Almasy, L.; Comuzzie, A.G.; Mahaney, M.C.; Jowett, J.B.M.; Shaw, J.; et al. Plasma lipid profiling in a large population-based cohort. J. Lipid Res. 2013, 54, 2898-2908. [CrossRef] [PubMed]

27. Messner, B.; Bernhard, D. Smoking and cardiovascular disease: Mechanisms of endothelial dysfunction and early atherogenesis. Arterioscler. Thromb. Vasc. Biol. 2014, 34, 509-515. [CrossRef]

28. Craig WY, Palomaki GE, Haddow JE: Cigarette smoking and serum lipid and lipoprotein concentrations: An analysis of pub-lished data. BMJ 1989, 298, 784-788. [CrossRef] 
29. Ma, J.; Folsom, A.R.; Shahar, E.; Eckfeldt, J.H. Plasma fatty acid composition as an indicator of habitual dietary fat intake in mid-dle-aged adults. The Atherosclerosis Risk in Communities (ARIC) Study Investigators. Am. J. Clin. Nutr. 1995, 62, 564-571. [CrossRef]

30. Arab, L. Biomarkers of Fat and Fatty Acid Intake. J. Nutr. 2003, 133 (Suppl. S3), 925S-932S. [CrossRef] [PubMed]

31. Shahin, M.H.; Gong, Y.; Frye, R.F.; Rotroff, D.M.; Beitelshees, A.L.; Baillie, R.A.; Chapman, A.B.; Gums, J.G.; Turner, S.T.; Boerwinkle, E.; et al. Sphingolipid Metabolic Pathway Impacts Thiazide Diuretics Blood Pressure Response: Insights from Genomics, Metabolomics, and Lipidomics. J. Am. Heart Assoc. 2018, 7, e006656. [CrossRef] [PubMed]

32. Zheng, H.; Xie, X.; Xie, N.; Xu, H.; Huang, J.; Luo, M. Sphingomyelin levels in nondipper and dipper hypertensive patients. Exp. Ther. Med. 2014, 7, 599-603. [CrossRef]

33. Fernandez, C.; Sandin, M.; Sampaio, J.; Almgren, P.; Narkiewicz, K.; Hoffmann, M.; Hedner, T.; Wahlstrand, B.; Simons, K.; Shevchenko, A.; et al. Plasma Lipid Composition and Risk of Developing Cardiovascular Disease. PLoS ONE 2013, 8, e71846. [CrossRef] [PubMed]

34. Meikle, P.J.; Wong, G.; Barlow, C.; Weir, J.M.; Greeve, M.A.; MacIntosh, G.L.; Almasy, L.; Comuzzie, A.G.; Mahaney, M.C.; Kowalczyk, A.; et al. Plasma Lipid Profiling Shows Similar Associations with Prediabetes and Type 2 Diabetes. PLoS ONE 2013, 8, e74341. [CrossRef]

35. Lu, J.; Lam, S.M.; Wan, Q.; Shi, L.; Huo, Y.; Chen, L.; Tang, X.; Li, B.; Wu, X.; Peng, K.; et al. High-Coverage Targeted Lipidomics Reveals Novel Serum Lipid Predictors and Lipid Pathway Dysregulation Antecedent to Type 2 Diabetes Onset in Normoglycemic Chinese Adults. Diabetes Care 2019, 42, 2117-2126. [CrossRef]

36. Sciacca, M.F.; Brender, J.R.; Lee, D.K.; Ramamoorthy, A. Phosphatidylethanolamine enhances amyloid fiber-dependent membrane fragmentation. Biochemistry 2012, 51, 7676-7684. [CrossRef] [PubMed]

37. Ference, B.A.; Graham, I.; Tokgozoglu, L.; Catapano, A.L. Impact of Lipids on Cardiovascular Health: JACC Health Promotion Series. J. Am. Coll. Cardiol. 2018, 72, 1141-1156. [CrossRef]

38. Shahidi, F.; Ambigaipalan, P. Omega-3 Polyunsaturated Fatty Acids and Their Health Benefits. Annu. Rev. Food Sci. Technol. 2018, 9, 345-381. [CrossRef]

39. Bazinet, R.P.; Layé, S. Polyunsaturated fatty acids and their metabolites in brain function and disease. Nat. Rev. Neurosci. 2014, 15, 771-785. [CrossRef]

40. Jha, P.; McDevitt, M.T.; Halilbasic, E.; Williams, E.; Quiros, P.M.; Gariani, K.; Sleiman, M.B.; Gupta, R.; Ulbrich, A.; Jochem, A.; et al. Genetic Regulation of Plasma Lipid Species and Their Association with Metabolic Phenotypes. Cell Syst. 2018, 6, 709-721.e6. [CrossRef]

41. Monnerie, S.; Comte, B.; Ziegler, D.; Morais, J.A.; Pujos-Guillot, E.; Gaudreau, P. Metabolomic and Lipidomic Signatures of Metabolic Syndrome and its Physiological Components in Adults: A Systematic Review. Sci. Rep. 2020, 10, 669. [CrossRef]

42. Jiang, X.-C.; Paultre, F.; Pearson, T.A.; Reed, R.G.; Francis, C.K.; Lin, M.; Berglund, L.; Tall, A.R. Plasma Sphingomyelin Level as a Risk Factor for Coronary Artery Disease. Arter. Thromb. Vasc. Biol. 2000, 20, 2614-2618. [CrossRef]

43. Van der Veen, J.N.; Kennelly, J.P.; Wan, S.; Vance, J.E.; Vance, D.E.; Jacobs, R.L. The critical role of phosphatidylcholine and phospha-tidylethanolamine metabolism in health and disease. Biochim. Biophys. Acta Biomembr. 2017, 1859, 1558-1572. [CrossRef]

44. Hatch, G.M.; Karmin, O.; Choy, P.C. Regulation of phosphatidylcholine metabolism in mammalian hearts. Biochem. Cell Biol. 1989, 67, 67-77. [CrossRef]

45. Vance, D.E. Role of phosphatidylcholine biosynthesis in the regulation of lipoprotein homeostasis. Curr. Opin. Lipidol. 2008, 19, 229-234. [CrossRef]

46. Bar-On, H.; Roheim, P.S.; Stein, O.; Stein, Y. Contribution of floating fat triglyceride and of lecithin towards formation of secretory triglyceride in perfused rat liver. Biochim. Biophys. Acta 1971, 248, 1-11. [CrossRef]

47. Tran, K.; Sun, F.; Cui, Z.; Thorne-Tjomsland, G.; St Germain, C.; Lapierre, L.R.; McLeod, R.S.; Jamieson, J.C.; Yao, Z. Attenuated secretion of very low density lipoproteins from McA-RH7777 cells treated with eicosapentaenoic acid is associated with impaired utilization of triacylglycerol synthesized via phospholipid remodeling. Biochim. Biophys. Acta 2006, 1761, 463-473. [CrossRef] [PubMed]

48. Makide, K.; Uwamizu, A.; Shinjo, Y.; Ishiguro, J.; Okutani, M.; Inoue, A.; Aoki, J. Novel lysophosphoplipid receptors: Their structure and function. J. Lipid Res. 2014, 55, 1986-1995. [CrossRef] [PubMed]

49. Sekas, G.; Patton, G.M.; Lincoln, E.C.; Robins, S.J. Origin of plasma lysophosphatidylcholine: Evidence for direct hepatic secretion in the rat. J. Lab. Clin. Med. 1985, 105, 190-194.

50. Iqbal, J.; Walsh, M.T.; Hammad, S.; Hussain, M.M. Sphingolipids and Lipoproteins in Health and Metabolic Disorders. Trends Endocrinol. Metab. 2017, 28, 506-518. [CrossRef] [PubMed]

51. Nilsson, Å.; Duan, R.-D. Absorption and lipoprotein transport of sphingomyelin. J. Lipid Res. 2006, 47, 154-171. [CrossRef] [PubMed]

52. Augé, N.; Escargueil-Blanc, I.; Lajoie-Mazenc, I.; Suc, I.; Andrieu-Abadie, N.; Pieraggi, M.-T.; Chatelut, M.; Thiers, J.-C.; Jaffrézou, J.-P.; Laurent, G.; et al. Potential Role for Ceramide in Mitogen-activated Protein Kinase Activation and Proliferation of Vascular Smooth Muscle Cells Induced by Oxidized Low Density Lipoprotein. J. Biol. Chem. 1998, 273, 12893-12900. [CrossRef] [PubMed]

53. Mantovani, A.; Dugo, C. Ceramides and risk of major adverse cardiovascular events: A meta-analysis of longitudinal studies. $J$. Clin. Lipidol. 2020, 14, 176-185. [CrossRef] [PubMed] 
54. Yao, K.; Wang, Y.; Xu, D.; Liu, X.; Shen, C.; Hu, W.; Wang, Z.; Wu, R.; Tang, X.; Sun, A.; et al. Effect of combined testing of ceramides with high-sensitive troponin $\mathrm{T}$ on the detection of acute coronary syndrome in patients with chest pain in China: $\mathrm{A}$ prospective observational study. BMJ Open 2019, 9, e028211. [CrossRef]

55. Gao, X.; Ke, C.; Liu, H.; Liu, W.; Li, K.; 599 Yu, B.; Sun, M. Large-scale Metabolomic Analysis Reveals Potential Biomarkers for Early Stage Coronary Atherosclerosis. Sci. Rep. 2017, 7, 11817. [CrossRef]

56. Sigruener, A.; Kleber, M.; Heimerl, S.; Liebisch, G.; Schmitz, G.; Maerz, W. Glycerophospholipid and Sphingolipid Species and Mortality: The Ludwigshafen Risk and Cardiovascular Health (LURIC) Study. PLoS ONE 2014, 9, e85724. [CrossRef]

57. Yin, X.; Willinger, C.M.; Keefe, J.; Liu, J.; Fernández-Ortiz, A.; Ibanez, B.; Peñalvo, J.; Adourian, A.; Chen, G.; Corella, D.; et al. Lipidomic profiling identifies signatures of metabolic risk. EBioMedicine 2020, 51, 102520. [CrossRef]

58. Forget, G.; Doyon, M.; Lacerte, G.; Labonté, M.; Brown, C.; Carpentier, A.C.; Langlois, M.-F.; Hivert, M.-F. Adoption of American Heart Association 2020 Ideal Healthy Diet Recommendations Prevents Weight Gain in Young Adults. J. Acad. Nutr. Diet. 2013, 113, 1517-1522. [CrossRef]

59. Elliott, P.; Peakman, T.C.; Biobank, O.B.O.U. The UK Biobank sample handling and storage protocol for the collection, processing and archiving of human blood and urine. Int. J. Epidemiol. 2008, 37, 234-244. [CrossRef]

60. Folch, J.; Lees, M.; Sloane Stanley, G.H. A Simple Method for the Isolation and Purification of Total Lipides from Animal Tissues. J. Biol. Chem. 1957, 226, 497-509. [CrossRef]

61. Rivas Serna, I.M.; Romito, I.; Maugeri, A.; Lo Re, O.; Giallongo, S.; Mazzoccoli, G.; Oben, J.A.; Li Volti, G.; Mazza, T.; Alisi, A.; et al. A Lipidomic Signature Complements Stemness Features Acquisition in Liver Cancer Cells. Int. J. Mol. Sci. 2020, $21,8452$. [CrossRef] [PubMed] 